CASE REPORT

\title{
Transition of Sézary syndrome into mycosis fungoides after complete clinical and molecular remission under extracorporeal photophoresis
}

\author{
C Assaf, M Hummel, M Zemlin, M Steinhoff, C C Geilen, H Stein, C E Orfanos
}

J Clin Pathol 2004;57:1325-1328. doi: 10.1136/icp.2004.017160

Mycosis fungoides (MF) and Sézary syndrome (SS) are the most common clinical variants of cutaneous $T$ cell lymphoma. Although thought to be closely related to mature $T$ helper cells, the relation between the neoplastic cells in MF and SS is still not fully clarified. This report describes a patient with complete remission of SS under treatment with extracorporeal photophoresis (ECP), who subsequently developed typical plaques of MF and large cell lymphoma (LCL). Serial polymerase chain reaction analyses confirmed identical $T$ cell receptor $\beta$ and $\gamma$ gene rearrangements in SS, MF, and $\mathrm{LCL}$, and complete disappearance of the circulating malignant $T$ cell clone from the peripheral blood after ECP. These findings indicate that the neoplastic cells in SS, MF, and $\mathrm{LCL}$ are derived from a common precursor $T$ cell, despite the change in clinical phenotype.

$\mathrm{P}$ imary cutaneous $\mathrm{T}$ cell lymphoma (CTCL) includes a heterogeneous group of lymphoproliferative disorders characterised by skin homing of malignant $\mathrm{T}$ cells. ${ }^{1}$ Mycosis fungoides (MF) and Sézary syndrome (SS) are the two major clinical variants of CTCL, both characterised by epidermotropic infiltrates of clonal malignant lymphocytes with cerebriform nuclei. ${ }^{2}$ SS shares many of the morphological and biological abnormalities of MF, including nuclear atypia, a mature $\mathrm{T}$ cell-like immunophenotype, frequent $\mathrm{T}$ cell receptor (TCR) gene rearrangements, and chromosomal aberrations, including PTEN gene abnormalities. ${ }^{3}$ However, SS shows some differences compared with MF, such as loss of the cutaneous lymphocyte antigen skin homing antigen that controls the ability of the malignant cells to circulate in the peripheral blood, and predominant secretion of $\mathrm{T}$ helper type 2 (Th2) cytokines. ${ }^{4}$ It is still a matter of controversy whether SS is simply a leukaemic phase of MF or a separate entity within the spectrum of CTCL.

"Mycosis fungoides and Sézary syndrome are the two major clinical variants of cutaneous T cell lymphoma, both characterised by epidermotropic infiltrates of clonal malignant lymphocytes with cerebriform nuclei"

Here, we describe a patient with SS, who initially experienced complete remission under extracorporeal photophoresis (ECP), but then developed plaques and nodular lesions of MF, with subsequent transformation into large cell lymphoma (LCL). To clarify whether the malignant $\mathrm{T}$ cells of SS, MF, and LCL were derived from the same neoplastic precursor or, alternatively, corresponded to independent $\mathrm{T}$ cell clones, we analysed their TCR $\beta$ and $\gamma$ rearrangements by means of the polymerase chain reaction (PCR), GeneScan analysis, and subsequent DNA sequencing, and found that the different clinical phenotypes resulted from a common neoplastic cell precursor.

\section{CASE REPORT}

A 72 year old man presented to the dermatology department, Charité-Universitätsmedizin, Berlin, Germany, with generalised erythroderma, palmar and plantar hyperkeratosis, enlarged lymph nodes, and recalcitrant itching (fig lA). The patient had raised white blood cell counts (between $27 \times 10^{9}$ /litre and $21 \times 10^{9}$ /litre) with large numbers of Sézary cells (32-52\%).

A skin biopsy revealed a moderately dense, perivascular lymphohistiocytic infiltrate in the upper dermis, with single cell epidermotropism of atypical lymphocytes showing hyperchromatic, convoluted nuclei (fig ID). Histological examination of a palpable lymph node showed infiltration of atypical small and large lymphocytes with a cerebriform appearance. Serological studies for human T lymphotropic virus 1 and human immunodeficiency virus were negative. Flow cytometric immunophenotyping of peripheral blood lymphocytes revealed a $\mathrm{CD} 3^{+} \mathrm{CD} 4^{+} \mathrm{CD} 7^{-} \mathrm{CD} 8^{-}$immunophenotype. Clonal TCR $\beta$ and $\gamma$ rearrangements of identical size and sequence were detected in all specimens from the skin, lymph node, and the peripheral blood. ${ }^{5-7}$ Further investigations excluded involvement of visceral organs or the bone marrow. All diagnostic criteria for typical SS according to the current World Health Organisation classification ${ }^{8}$ and the consensus conference of the International Society for Cutaneous Lymphomas ${ }^{9}$ were met.

Treatment with extracorporeal photophoresis, comprising two consecutive sessions every four weeks, resulted in complete resolution of the skin symptoms after six months, including remission of the lymph node enlargement and disappearance of the circulating Sézary cells. Furthermore, clonal TCR $\beta$ and $\gamma$ gene rearrangements were no longer detectable in the peripheral blood, indicating that the malignant $\mathrm{T}$ cell clone had disappeared. However, three months later the patient developed typical plaques and papulonodular lesions on his trunk (fig 1B). Histopathological examination showed features of MF with a band-like infiltrate in the upper dermis, atypical $\mathrm{T}$ cells with cerebriform nuclei, and the formation of intraepidermal microabcesses (Pautrier abscesses) (fig 1E). No extracutaneous involvement was found, including peripheral blood, where atypical cells were absent. In addition, the patient received 35 phototherapy sessions (psoralen and ultraviolet A irradiation of the skin) leading to major clinical improvement.

Abbreviations: $\mathrm{CTCL}$, cutaneous T cell lymphoma; $\mathrm{ECP}$, extracorporeal photophoresis; LCL, large cell lymphoma; MF, mycosis fungoides; PCR, polymerase chain reaction; SS, Sézary syndrome; TCR, T cell receptor; Th, T helper cell 


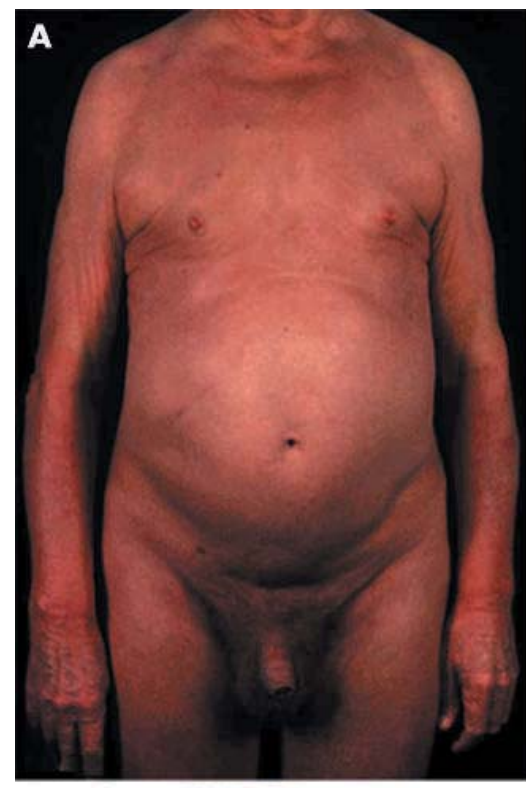

D

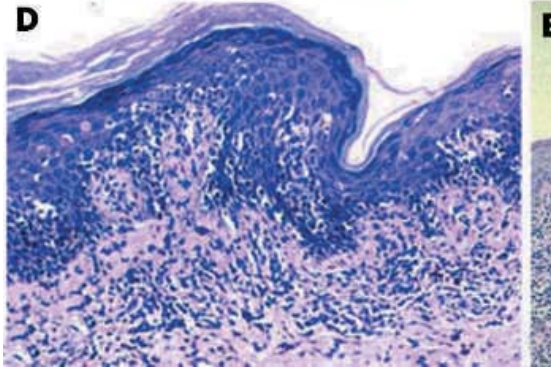

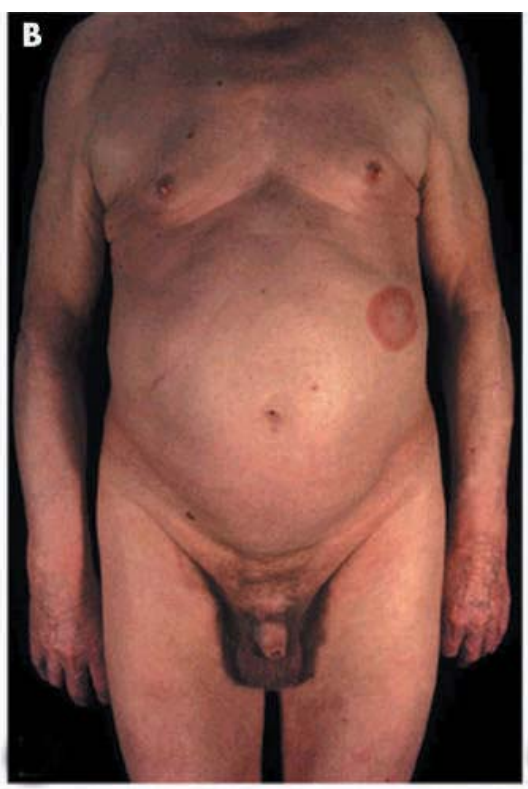

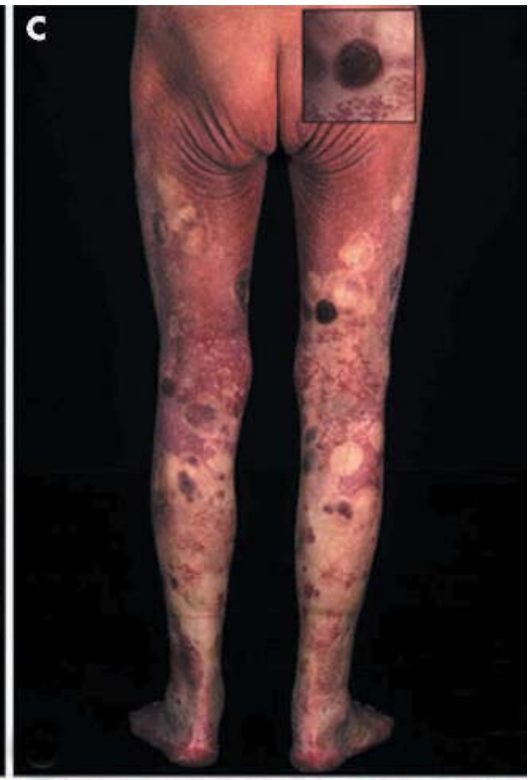

E

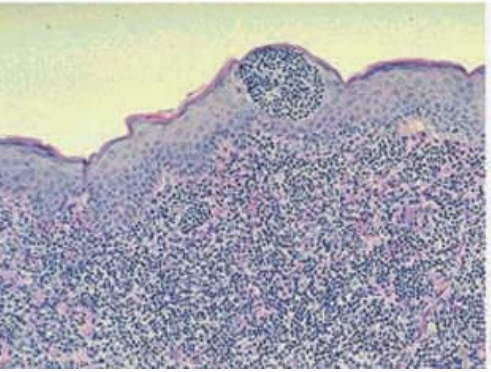

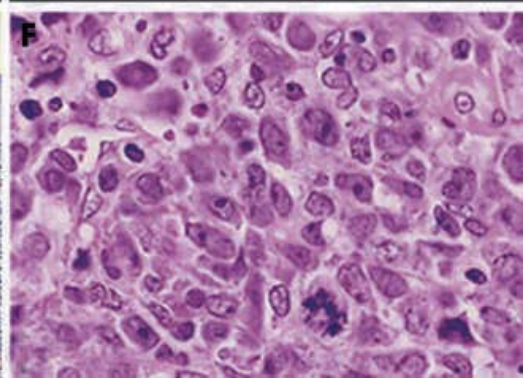

Figure 1 (A) Typical erythroderma of Sézary syndrome, and (B) solitary mycosis fungoides plaque, (C) tumour nodule of large cell lymphoma (LCL). Histology reveals (D) an upper band-like infiltrate with epidermotropism of atypical lymphocytes and (E) a typical Pautrier microabscess (E). (F) LCL showing sheets of blast cells.

Seventeen months later the patient developed progressive disease with multiple skin tumours, generalised lymphadenopathy, and infiltration of the bone marrow, liver, and tonsils (fig IC). Histology revealed large cell transformation of the tumour cells $\left(\mathrm{CD} 30^{-}\right.$) (fig IF). Despite introduction of electron beam skin radiation and polychemotherapy (five cycles of CHOP regimen) the patient died, two and half years after the first diagnosis of SS.

\section{RESULTS AND DISCUSSION}

Although MF and SS are generally thought to belong to the group of malignant CTCLs, their relation to each other has

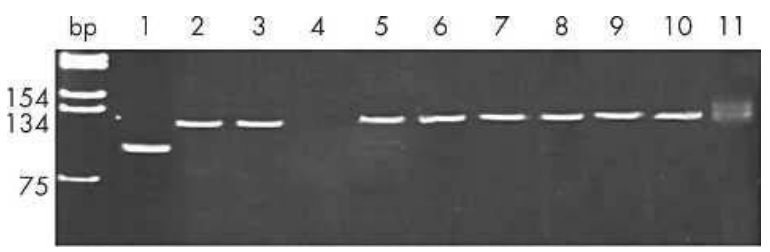

Figure 3 T cell receptor $\beta$ polymerase chain reaction $16 \%$ polyacrylamide gel stained with ethidium bromide). Lane 1, positive control T cell line Hut; lane 2, skin in the Sézary syndrome (SS) phase; lane 3, blood in the SS phase; lane 4, blood after six cycles of extracorporeal photophoresis; lanes 5 and 6 , skin in the mycosis fungoides (MF) phase; lane 7, lymph node in the large cell lymphoma (LCL) phase; lane 8, bone marrow in the LCL phase; lane 9, spleen in the LCL phase; lane 10, tonsil in the LCL phase; lane 11, negative control. $T$ cell clones as the origin of the different cutaneous $T$ cell lymphoma phenotypes. 
not yet been fully clarified. Patients with MF, who comprise $70 \%$ of all cases of CTCL, normally present with cutaneous patches and plaques and pursue a longterm, indolent clinical course..$^{1-3}{ }^{10}$ However, in some patients there is a progression to erythrodermic disease, which may be associated with morphological changes in the peripheral blood. In contrast, SS is a leukaemic variant of CTCL, clinically characterised by early onset of erythroderma, pruritus, and peripheral lymphadenopathy. It is an aggressive clinical entity associated with poor prognosis and median survival of two to three years. ${ }^{1-3}$ In rare cases, MF can evolve into SS during progression of the disease. ${ }^{2}$ In addition, the incidence of large cell transformation in MF and less often in SS is a well known phenomenon, having been reported in $8 \%$ to $55 \%$ of cases. ${ }^{11-14}$ However, to our knowledge, the transition of SS into MF has not been reported yet.

There are three possible explanations of the unusual change in the clinical phenotype in our patient, namely: (1) the established SS progressed smoothly into MF and was then subsequently transformed into LCL; (2) SS and MF developed separately from a common precursor cell, with subsequent transformation of the MF into LCL; or (3) SS, MF, and LCL each arose separately from unrelated T cell clones (fig 2). To investigate the validity of these possibilities, we compared the rearrangement of the TCR genes in the three CTCL phenotypes, as related to the clinical course.

Clonal TCR rearrangements of the same size were detected in skin, blood, and lymph node at the initial diagnosis of SS, as shown by GeneScan analysis of the TCR $\beta$ and $\gamma$ PCR products. During clinical remission, no clonal T cell populations were detected in the peripheral blood. However, a T cell clone identical to that of SS could be identified in early MF lesions and during the LCL phase in lymph node, spleen, tonsil, and bone marrow (fig 3). Sequence analysis demonstrated that the clonal PCR products were not only of the same size but also had the same sequence (V $\beta 19 D 2 J \beta 2.7)$, thus demonstrating the identity of the clonal $\mathrm{T}$ cell populations involved.

"The presence of the same T cell receptor rearrangements in the neoplastic cells from Sézary syndrome, mycosis fungoides, and large cell lymphoma found in our patient show that they were derived from a common T cell clone"

These results rule out the possibility that the clonal $\mathrm{T}$ cell populations of the three clinical entities originated from different or unrelated precursors. The presence of the same TCR rearrangements in the neoplastic cells from SS, MF, and LCL found in our patient show that they were derived from a common $\mathrm{T}$ cell clone. The clinical development of different lymphoma phenotypes harbouring the identical cell clone has already been described in B cell lymphoma (for example, Hodgkin disease in combination with follicular lymphoma), ${ }^{15}$ and in T cell lymphoproliferations, such as MF or anaplastic large cell lymphoma in combination with lymphomatoid papulosis. ${ }^{16-18}$

The complete remission of the SS seen in our patient may have been the result of his good response to ECP. In addition to the low side effect profile of ECP, this treatment modality has been shown to be effective and to induce complete remission in several patients with SS. ${ }^{19-22}$ Recent findings indicate that ECP induces a clone specific Th-1 type immune response, with subsequent selective reduction of the malignant $\mathrm{T}$ cell population, as a result of $\mathrm{T}$ cell apoptosis and the modulation of dendritic cell differentiation. ${ }^{192324}$ These findings were confirmed in our patient by the complete disappearance of circulating blood tumour cells and the complete regression of erythroderma after ECP treatment.
Take home messages

- We describe a patient with complete remission of Sézary syndrome (SS) under treatment with extracorporeal photophoresis (ECP), who subsequently developed typical plaques of mycosis fungoides (MF) and large cell lymphoma (LCL)

- Serial polymerase chain reaction analysis confirmed identical T cell receptor $\beta$ and $\gamma$ gene rearrangements in $\mathrm{SS}, \mathrm{MF}$, and $\mathrm{LCL}$, and complete disappearance of the circulating malignant $T$ cell clone from the peripheral blood after ECP

- These findings indicate that the neoplastic cells in SS, $M F$, and $L C L$ are derived from a common precursor $T$ cell, despite the change in the clinical phenotype

The underlying reasons for the relapse with a clinical picture of MF are unknown. However, the "pro Th-1 milieu" created by the ECP could have altered the activation pattern of the low number of surviving SS cells towards MF cells. ${ }^{25}$

To our knowledge, this is the first report describing the transition of SS into MF. The neoplastic cells of the SS and the MF found in our patient were derived from a common T cell, which finally progressed to an LCL phenotype. Therefore, it seems reasonable to assume that SS, MF, and LCL represent a common genotype with different possible phenotypes of cutaneous lymphoma.

\section{ACKNOWLEDGEMENTS}

We are grateful to $\mathrm{H}-\mathrm{H}$ Müller for his excellent technical assistance.

\section{Authors' affiliations}

C Assaf, M Steinhoff, C C Geilen, C E Orfanos, Department of Dermatology, Charité-Universitätsmedizin Berlin, Campus Benjamin Franklin, Fabeckstrasse 60-62, 14195 Berlin, Germany

M Hummel, H Stein, Institute of Pathology, Charité-Universitätsmedizin Berlin

M Zemlin, Department of Neonatology and Neuropediatrics, Philipps University, Deutschhausstr. 12, 35037 Marburg, Germany

Correspondence to: Dr C Assaf, Department of Dermatology, CharitéUniversitätsmedizin Berlin, Campus Benjamin Franklin, Fabeckstrasse 60-62, 14195 Berlin, Germany; chalid.assa@@charite.de

Accepted for publication 1 June 2004

\section{REFERENCES}

1 Siegel RS, Pandolfino T, Guitart J, et al. Primary cutaneous T-cell lymphoma: review and current concepts. J Clin Oncol 2000;18:2908-25.

2 Kim YH, Liu HL, Mraz-Gernhard S, et al. Long-term outcome of 525 patients with mycosis fungoides and Sézary syndrome: clinical prognostic factors and risk for disease progression. Arch Dermatol 2003;139:857-66.

$3 \mathrm{Kim}$ YH, Hoppe RT. Mycosis fungoides and the Sézary syndrome. Semin Oncol 1999:26:276-89.

4 Saed G, Fivenson DP, Naidu Y, et al. Mycosis fungoides exhibits a Th1-type cell-mediated cytokine profile whereas Sézary syndrome expresses a Th2-type profile. J Invest Dermatol 1994;103:29-33.

5 Zemlin M, Hummel M, Anagnostopoulos I, et al. Improved polymerase chain reaction detection of clonally rearranged T-cell receptor beta chain genes. Diagn Mol Pathol 1998;7:138-45.

6 Assaf C, Hummel M, Dippel E, et al. High detection rate of T-cell receptor beta chain rearrangements in T-cell lymphoproliferations by family specific polymerase chain reaction in combination with the GeneScan technique and DNA-sequencing. Blood 2000;96:640-6.

7 Dippel E, Assaf C, Hummel M, et al. Clonal T-cell receptor $\gamma$-chain gene rearrangement by PCR-based GeneScan analysis in advanced cutaneous T-cell lymphoma: a critical evaluation. J Pathol 1999;188:146-54.

8 In: Jaffe ES, Harris NL, Stein H, Vardiman JW, eds. World Health Organisation classification of tumors. Pathology and genetics of tumors of haematopoietic and lymphoid tissues. Lyon: IARC Press, 2001. 
9 Vonderheid EC, Bernengo MG, Burg G, et al. Update on erythrodermic cutaneous T-cell lymphoma: report of the International Society for Cutaneous Lymphomas. J Am Acad Dermatol 2002;46:95-106.

10 Willemze R, Kerl H, Sterry W, et al. EORTC classification for primary cutaneous lymphomas: a proposal from the cutaneous lymphoma study group of the European Organization for Research and Treatment of Cancer. Blood 1997;90:354-71.

11 Diamandidou E, Colome-Grimmer M, Fayad L, et al. Transformation of mycosis fungoides/Sézary syndrome: clinical characteristics and prognosis Blood 1998;92:1150-9.

12 Kienitz T, Burg G, Schmoeckel C, et al. High-grade malignant lymphoma arising from Sézary's syndrome. A case report. Dermatologica 1979:158:126-36.

13 Sakai H, Matsuo S, Matsumoto $M$, et al. A case of Sézary syndrome associated with cutaneous transformation. J Dermatol 1996;23:697-702.

14 Carrozza PM, Kempf W, Kazakov DV, et al. A case of Sézary's syndrome associated with granulomatous lesions, myelodysplastic syndrome and transformation into CD30-positive large-cell pleomorphic lymphoma. Br J Dermatol 2002;147:582-6.

15 Marafioti T, Hummel M, Anagnostopoulos I, et al. Classical Hodgkin's disease and follicular lymphoma originating from the same germinal center B cell. $J$ Clin Oncol 1999; 17:3804-9.

16 Chott A, Vonderheid EC, Olbricht S, et al. The dominant T cell clone is present in multiple regressing skin lesions and associated T cell lymphomas of patients with lymphomatoid papulosis. J Invest Dermatol 1996;106:696-700.
17 Steinhoff M, Hummel M, Anagnostopoulos I, et al. Single-cell analysis of CD30+ cells in lymphomatoid papulosis demonstrates a common clonal T-cell origin. Blood 2002;100:578-84.

18 Assaf C, Hummel M, Dippel E, et al. Common clonal T-cell origin in a patient with T prolymphocytic leukaemia and associated cutaneous T-cell lymphomas. Br J Haematol 2003;120:488-91.

19 Macheiner W, Jantschitsch C, Graninger W, et al. Sézary syndrome and seronegative polyarthritis: treatment with extracorporeal photochemotherapy. J Am Acad Dermatol 2003;48:220-6.

20 Edelson R, Berger C, Gasparro F, et al. Treatment of cutaneous T-cell lymphoma by extracorporeal photochemotherapy. Preliminary results. N Engl J Med 1987;5:297-303.

21 Yoo EK, Cassin M, Lessin SR, et al. Complete molecular remission during biologic response modifier therapy for Sézary syndrome is associated with enhanced helper T type 1 cytokine production and natural killer cell activity. J Am Acad Dermatol 2001;45:208-16.

22 Dippel E, Goerdt S, Assaf C, et al. Cutaneous T-cell lymphoma severity index and T-cell gene rearrangement. Lancet 1997;350:1776-7.

23 Bladon J, Taylor PC. Extracorporeal photopheresis in cutaneous T-cell lymphoma and graft-versus-host disease induces both immediate and progressive apoptotic processes. Br J Dermatol 2002;146:59-68.

24 Tambur AR, Ortegel JW, Morales A, et al. Extracorporeal photopheresis induces lymphocyte but not monocyte apoptosis. Transplant Proc 2000:32:747-8.

25 Di Renzo M, Rubegni P, De Aloe G, et al. Extracorporeal photochemotherapy restores Th1/Th2 imbalance in patients with early stage cutaneous T-cell lymphoma. Immunology 1997;92:99-103. 